\title{
Health Information Risk Analysis Based on BMI Fluctuation
}

\author{
Yuki Takeyama, Katsunori Fujii \\ Graduate School of Business Administration and Computer Science, Aichi Institute of Technology, Aichi-prefecture, Japan \\ Email address: \\ yuki319takeyama@yahoo.co.jp (Y. Takeyama), fujii@aitech.ac.jp (K. Fujii)
}

\section{To cite this article:}

Yuki Takeyama, Katsunori Fujii. Health Information Risk Analysis Based on BMI Fluctuation. American Journal of Sports Science. Vol. 8, No. 4, 2020, pp. 105-110. doi: 10.11648/j.ajss.20200804.15

Received: December 1, 2020; Accepted: December 9, 2020; Published: December 22, 2020

\begin{abstract}
Obesity is a factor that lowers productivity in companies. In recent years there has been a focus on health management in the management of human resources, including obesity. Actually, as living habits change after people graduate from university and become company employees, a situation arises in which it is easy for obesity to occur from a lack of activity and the accumulation of stress. People therefore need to establish risk management for obesity while they are still university students. However, the concept of obesity as a human resource and the magnitude of that risk are not clear in university students. Since the cutoff value for obesity is not established, if health information on risk due to the degree of obesity were understood, it would perhaps contribute to the facilitation of health management in university students. In this study we assessed the level of health risk based on BMI fluctuations, calculated mean values for health information items for each unit of BMI for BMI values from 14 to 34, and analyzed fluctuations in health information items by analyzing changing trends in each item based on BMI fluctuation. The results showed that blood pressure and maximum oxygen uptake increased risks together with fluctuations in BMI. With this, it is thought that a new cutoff point for obesity risk can be established.
\end{abstract}

Keywords: Obesity, Health Information, BMI Fluctuation, Cutoff Value

\section{Introduction}

Although obesity is not a disease, it is known to carry many health risks. Within companies, obesity is a cause of decreased productivity and wage gaps. Furugouri [1] reported such findings in data from the USA. However, based on reports by the Japanese Ministry of Health, Labor and Welfare in Japan [2] and the National Center for Health Statistics (NCHS) in the USA [3], the obesity rate in Japan (26.3\%) is clearly lower than that in the USA $(39.6 \%)$. Moreover, considering that the standard for obesity in the USA is BMI $\geq 30$, while that in Japan is BMI $\geq 25$, it can be seen that the obesity rate in Japan is quite low. Thus, while the obesity rate in Japan is lower than that in western countries, considering the health risks of obesity this low level is still not necessarily good. A difference in the level of health between races exists in this difference. Matsumoto [4] explained the importance of aldehyde dehydrogenase 2 (ALDH2) polymorphisms, and since the activity of this gene is what makes Western people better able to break down alcohol, its activity in Asians would seem to be low. It is conjectured from this finding that Western people have a stronger resistance to toxicity than Asian. It may thus also be that the health risks of obesity are milder in Westerners than in Asians.

Be that as it may, there are still health risks of obesity among Asians. Asakawa [5] reported that four in ten people above the age of 40 are $10 \mathrm{~kg}$ or heavier than they were at age 20 . When people's living habits change after graduating from university and becoming a company employee, a situation arises in which it is easy for obesity to occur due to a lack of activity and the accumulation of stress. Therefore, it is important to learn measures to counter the risk obesity during the university years and attain health that can raise productivity as a company employee in the future. For that reason, there are calls for the establishment of risk management for obesity. This highlights the importance of anti-obesity measures. However, obesity is neither a disease nor necessarily unhealthy. Hayakawa and Fujii $[6,7]$ have proposed the concept of valid health and fitness, and suggested the importance for modern humans of having physical fitness suited to their respective living activities. In university students, the concept of obesity as a human resource and the magnitude of that health risk are not clear. Moreover, the fact that the cutoff value for obesity has not been established is thought to be one factor that is delaying the 
establishment of health management.

In conventional risk analyses of the effects on health from obesity, most have been done by analyzing the health risks from obesity by comparing health information in obese types classified as BMI of 25 to 30 or higher and standard types. If the health risk from one's degree of obesity could be analyzed, it should contribute greatly to the establishment of risk management for obesity in most people.

In this study, the subjects were healthy male university students with mature physiques. The level of obesity was ascertained based on BMI fluctuations, and the slide status of detailed figures in health information for each unit of BMI from BMI of 14 to 34 was clarified. An attempt was then made to establish a cutoff value for obesity with respect to health from the slide status of health information based on BMI fluctuations.

\section{Methods}

\subsection{Subjects and Measurements}

The subjects were 15,699 healthy male university students at a single university from 2008 to 2016 . The items measured were the physical composition items of height, weight, BMI, and body fat percentage as physical information, and systolic blood pressure, diastolic blood pressure, and maximal oxygen uptake, which are health information items. Subjects for whom all data for each item had been collected were used in the study.

\subsection{Analytical Method}

\subsubsection{Validity of Degree of Regression Polynomial}

In regression polynomials, regression lines from regression analysis with 2 variates are generally applied. However, there are cases with 2-variate regression analysis in which the second or higher order relations are judged to be more appropriate than first-order relations. In such cases, 2nd, 3rd, 4th, or other order approximation polynomials are applied. Matsuura and Kim [8] applied least squares approximation to height and weight growth, and investigated the growth pattern. Largo et al. [9] used spline smoothing to investigate height growth patterns. All of these methods apply least squares approximation polynomials, and have led to useful findings. From these reports, an appropriate order for the regression polynomial of health information against BMI was determined in this study based on an investigation of the residual sum of squares that was applied by Matsuura and Kim [8].

\subsubsection{Wavelet Interpolation Method (WIM)}

To describe an approximation of the true growth curve from the obtained growth data, a wavelet function was used for interpolation between data. Growth distance value curves were drawn and those distance value curves were differentiated to derive the obtained growth velocity curve. Figure 1 showed this. The age at maximum peak velocity
(MPV) in growth during puberty was identified. In this study, only the growth distance value curves are used. The characteristics of the wavelet interpolation model are that local events can be sensitively read, and the accuracy of approximations is very high. The theoretical background and grounds for its efficacy have been described in previous reports (Fujii and Yamamoto; Fujii and Matsuura; Fujii and Demura [10-12]).

In this study, by applying the wavelet interpolation method to least square approximation polynomials for the constructed health information items, finer changes could be read and the detailed trends in health information risk in BMI fluctuations could be analyzed. From that, more accurate critical points and inflection points were calculated.

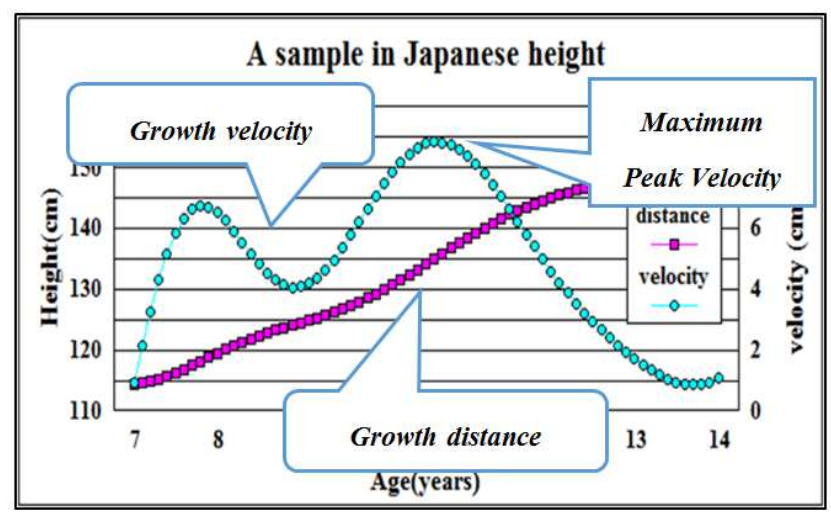

Figure 1. Wavelet interpolation method (Sample).

\subsection{Analytical Procedures}

1) Subjects with BMI from 14 to 34 were selected and mean values for health information items were calculated for each unit of BMI.

2) A polynomial regression analysis was performed with mean values for the calculated health information items and BMI, and least squares approximation polynomials were constructed.

3) The wavelet interpolation method was applied to the constructed least squares approximation polynomials and the health information risk trends with changes in BMI for each health information item were analyzed.

4) From the analysis results, cutoff values for each health information item were sought.

\section{Results}

\subsection{Fundamental Statistics}

All items showed subtle changes in health risk with respect to BMI fluctuations. The number of subjects showed normal distributions for all items with a BMI of 19 as the boundary. Also, there was no significant variation in the standard deviation of all BMI values for all items. (Table 1) 
Table 1. Fundamental statistics of body fat percentage and health information items.

\begin{tabular}{|c|c|c|c|c|c|c|c|c|}
\hline \multirow{2}{*}{ BMI } & \multicolumn{2}{|c|}{ Body fat percentage } & \multicolumn{2}{|c|}{ Systolic blood pressure } & \multicolumn{2}{|c|}{ Diastolic blood pressure } & \multicolumn{2}{|c|}{ Maximal oxygen uptake } \\
\hline & Mean \pm SD & $\mathbf{N}$ & $\operatorname{Mean} \pm$ SD & $\mathbf{N}$ & Mean \pm SD & $\mathbf{N}$ & Mean \pm SD & $\mathbf{N}$ \\
\hline 14 & $8.12 \pm 1.36$ & 26 & $115.50 \pm 14.79$ & 26 & $73.65 \pm 9.26$ & 26 & $44.38 \pm 9.25$ & 25 \\
\hline 15 & $9.39 \pm 1.92$ & 142 & $115.71 \pm 13.78$ & 144 & $70.42 \pm 10.02$ & 142 & $47.34 \pm 9.37$ & 137 \\
\hline 16 & $10.47 \pm 2.02$ & 530 & $114.91 \pm 12.33$ & 529 & $69.94 \pm 9.39$ & 530 & $48.40 \pm 9.74$ & 520 \\
\hline 17 & $11.93 \pm 2.30$ & 1196 & $115.90 \pm 12.85$ & 1209 & $69.55 \pm 9.53$ & 1196 & $47.79 \pm 9.09$ & 1199 \\
\hline 18 & $13.22 \pm 2.51$ & 1983 & $115.62 \pm 12.30$ & 1992 & $68.78 \pm 9.15$ & 1983 & $48.70 \pm 8.84$ & 1972 \\
\hline 19 & $14.66 \pm 2.59$ & 2355 & $117.03 \pm 12.79$ & 2369 & $68.18 \pm 9.46$ & 2355 & $48.56 \pm 9.23$ & 2354 \\
\hline 21 & $17.41 \pm 3.09$ & 1918 & $119.15 \pm 12.44$ & 1932 & $68.43 \pm 9.20$ & 1918 & $47.71 \pm 9.03$ & 1916 \\
\hline 22 & $18.83 \pm 3.21$ & 1449 & $120.71 \pm 12.70$ & 1458 & $69.04 \pm 9.26$ & 1449 & $47.04 \pm 9.39$ & 1443 \\
\hline 23 & $20.31 \pm 3.64$ & 1095 & $121.00 \pm 13.46$ & 1094 & $69.53 \pm 9.43$ & 1095 & $45.10 \pm 9.49$ & 1095 \\
\hline 24 & $21.85 \pm 3.55$ & 767 & $123.68 \pm 13.31$ & 768 & $70.41 \pm 9.51$ & 767 & $44.13 \pm 9.07$ & 760 \\
\hline 25 & $23.60 \pm 3.88$ & 472 & $125.51 \pm 12.88$ & 471 & $71.61 \pm 9.85$ & 472 & $42.83 \pm 8.70$ & 470 \\
\hline 26 & $24.77 \pm 4.02$ & 341 & $125.63 \pm 13.87$ & 337 & $72.68 \pm 10.11$ & 341 & $41.91 \pm 9.79$ & 335 \\
\hline 27 & $26.68 \pm 3.57$ & 229 & $128.98 \pm 14.24$ & 223 & $74.45 \pm 10.07$ & 229 & $41.05 \pm 9.07$ & 230 \\
\hline 28 & $27.52 \pm 3.48$ & 166 & $127.88 \pm 12.65$ & 162 & $73.97 \pm 9.36$ & 166 & $39.45 \pm 7.93$ & 164 \\
\hline 30 & $29.14 \pm 4.73$ & 88 & $131.38 \pm 13.82$ & 86 & $74.73 \pm 10.69$ & 88 & $35.78 \pm 7.70$ & 90 \\
\hline 31 & $29.32 \pm 5.00$ & 66 & $130.68 \pm 15.07$ & 66 & $77.21 \pm 10.35$ & 66 & $36.55 \pm 7.29$ & 69 \\
\hline 32 & $30.27 \pm 3.51$ & 47 & $132.78 \pm 15.78$ & 46 & $77.68 \pm 10.24$ & 47 & $36.99 \pm 9.20$ & 47 \\
\hline 33 & $30.10 \pm 6.16$ & 34 & $136.24 \pm 15.02$ & 34 & $79.09 \pm 11.70$ & 34 & $35.77 \pm 6.68$ & 33 \\
\hline 34 & $32.03 \pm 2.61$ & 18 & $139.44 \pm 10.95$ & 18 & $77.39 \pm 8.17$ & 18 & $33.38 \pm 5.70$ & 18 \\
\hline
\end{tabular}

\subsection{Polynomial Regression Analysis and Construction of Least Squares Approximation Polynomial}

The numerical value of the coefficient of determination is high in each item, and it is considered that a reasonable least squares approximation polynomial is obtained. (Figures 2, 3, 4, 5)

\subsection{Trends in Changes of Physique Information Items (Body Fat Percentage) with BMI Fluctuations}

The change in body fat percentage accompanying BMI fluctuation always tended to rise. There are disadvantages to judging obesity with BMI, when even people with much muscle and little body fat are judged to be obese. However, in the results of this study, body fat percentage tended to rise as BMI increased. Therefore, the subjects in this study were thought to be judged to be obese based on increased body fat. (Figure 6)

\subsection{Trends in Changes of Health Information Items with BMI Fluctuations}

The change trend in systolic blood pressure with BMI fluctuations was always a rising trend. In addition, because systolic blood pressure always showed a rising trend, no critical point was seen. However, an inflection point was seen when BMI was 29.6 (Figure 7). The change trend in diastolic blood pressure with BMI fluctuation was seen to be a falling trend until BMI was 19.1. A BMI of 19.1 was taken to be a critical point, and after that critical point diastolic blood pressure showed a rising trend (Figure 8 ). The change trend in maximal blood pressure with fluctuation in BMI was seen to be a rising trend until a BMI of 17.6, which was taken to be the critical point. Beyond that critical point, maximal blood pressure showed a falling trend (Figure 9)

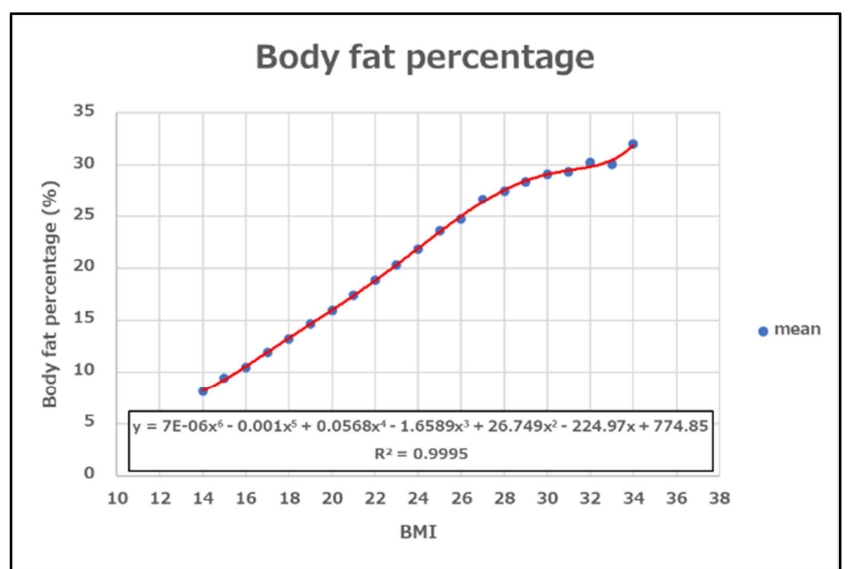

Figure 2. Polynomial regression analysis on body fat percentage and construction of least squares approximation polynomial.

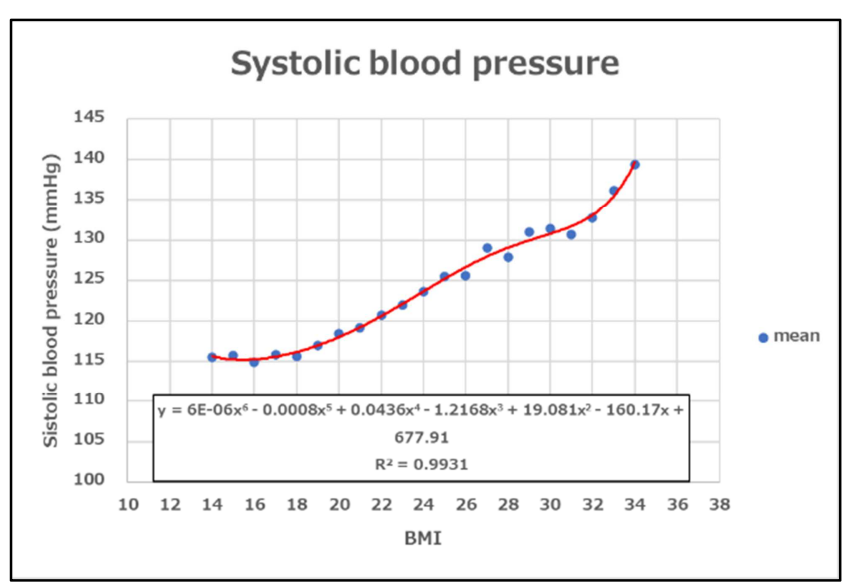

Figure 3. Polynomial regression analysis on systolic blood pressure and construction of least squares approximation polynomial. 


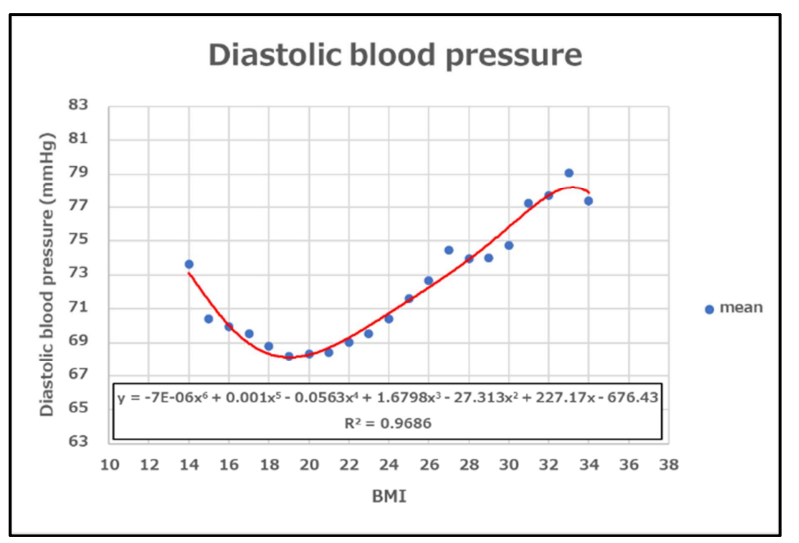

Figure 4. Polynomial regression analysis on diastolic blood pressure and construction of least squares approximation polynomial.

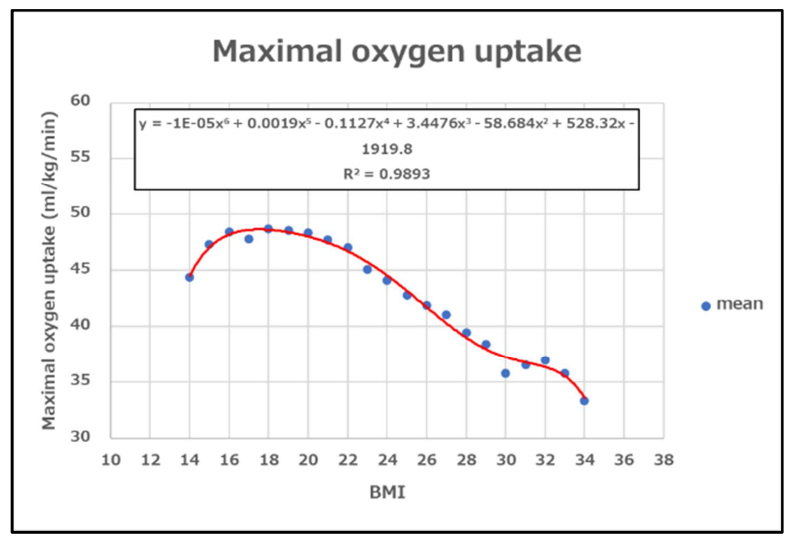

Figure 5. Polynomial regression analysis on maximal oxygen uptake and construction of least squares approximation polynomial.

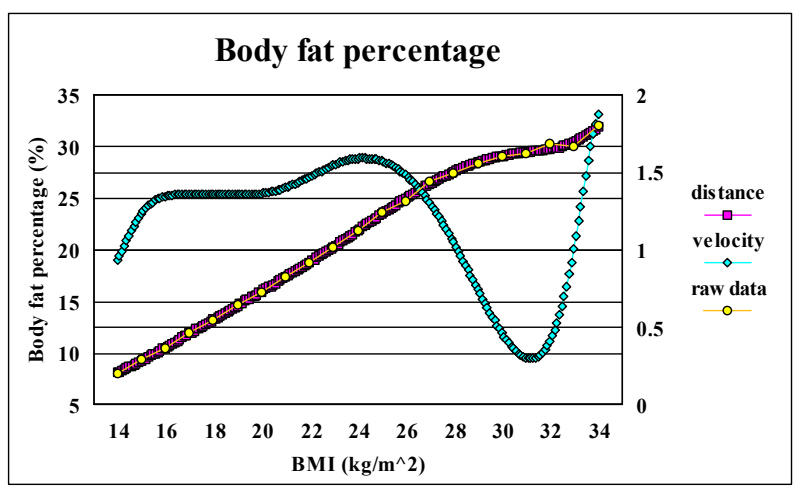

Figure 6. Change trend of Body fat percentage by BMI fluctuation.

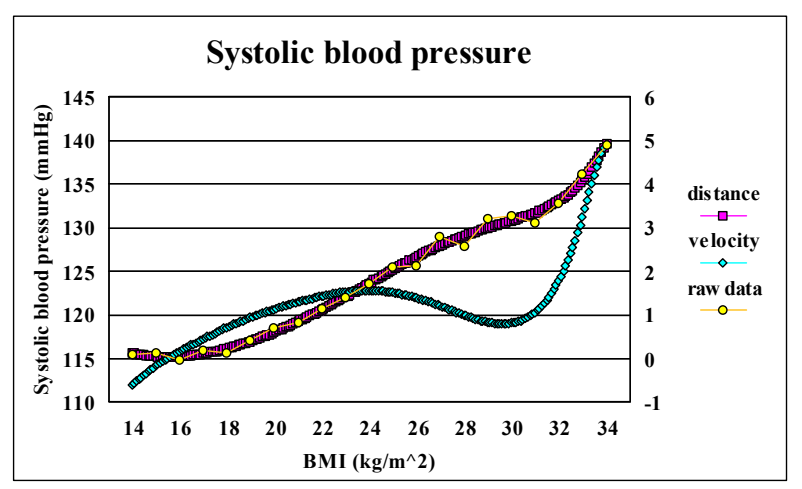

Figure 7. Change trend of Systolic blood pressure by BMI fluctuation.

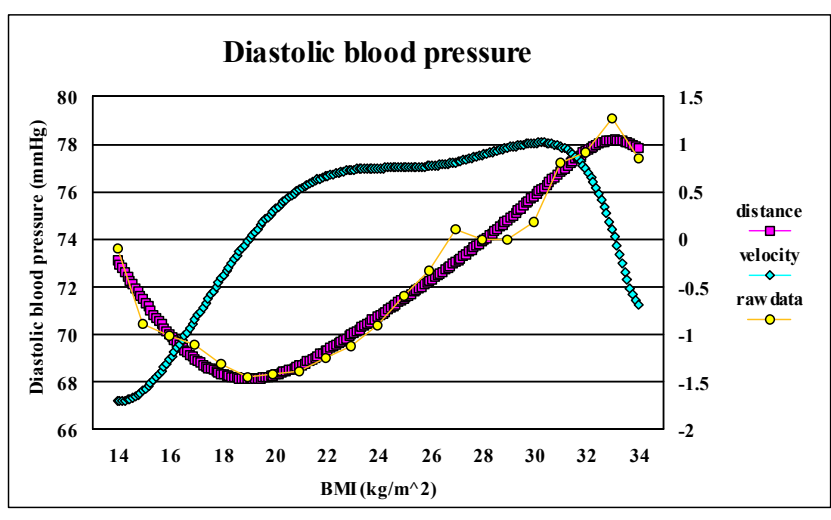

Figure 8. Change trend of Diastolic blood pressure by BMI fluctuation.

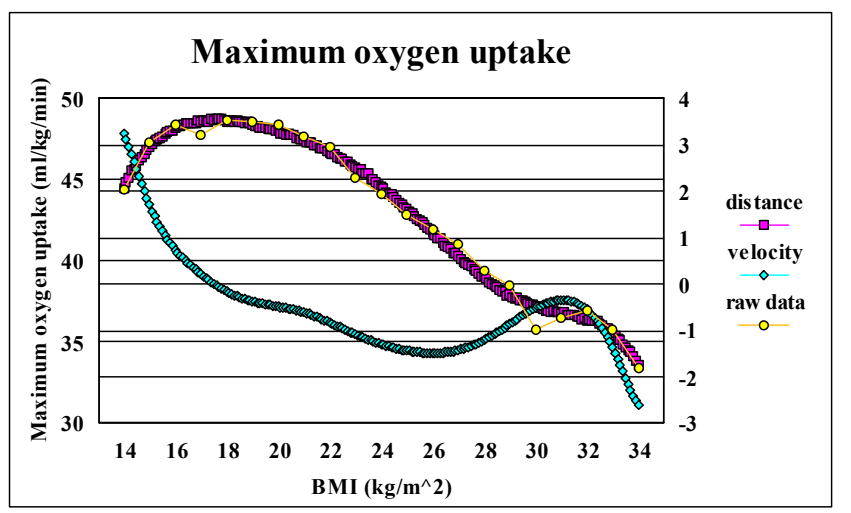

Figure 9. Change trend of Maximum oxygen uptake by BMI fluctuation.

\section{Discussion}

Body mass index (BMI) is an index of physique derived from the finding by Quetelet [13] in French people that body weight is proportional to the square of height. Later, Key [14] showed a high correlation between BMI and body fat percentage, which led to the positioning of BMI as an index of fatness today. Thus, BMI is understood to reflect body fat percentage with considerable accuracy. However, in recent years bioelectrical impedance analysis (BIA) has been used to calculate the sum of subcutaneous fat and visceral fat as a percentage of body weight. Consequently, specification of visceral fat, which is considered to be a factor in lifestyle-related diseases, has become ambiguous. The BIA method used in this study is also calculated with just the sum of subcutaneous fat and visceral fat, and analyzing the strict relationship with disease is difficult. Adopting simple BMI gives the advantage of being able to analyze large amounts of data.

In addition, in recent years the diagnostic criteria for metabolic syndrome include not body fat volume (percentage), but waist circumference. This is a standard derived from the high correlation between visceral fat and waist circumference [15] to obtain simplicity. However, waist circumference also has a high correlation with BMI unrelated to gender differences [16]. Kuroki [17] proposed the BMI calculation formula below and derived a close relationship with waist circumference, and pointed out a close relationship between metabolic syndrome and BMI. 


$$
\begin{gathered}
\text { BMI }=\frac{\text { Weight }(\mathrm{kg})}{\text { Height }(\mathrm{m})^{2}} \propto \frac{\text { Height } \times \text { Cross sectional area }}{\text { Height } \times \text { Height }} \propto \frac{\text { Height } \times \text { Waist circumference } \times \text { Waist circumference }}{\text { Height } \times \text { Height }} \\
=\frac{(\text { Waist circumference })^{2}}{\text { Height }}
\end{gathered}
$$

The reason BMI was adopted in this study, therefore, was because it was thought that if a standard for fatness could be established with a simple method, analysis of the risk of obesity could be easily performed.

In conventional risk analysis of the effects on health of obesity, BMI of 25 to 30 or higher is set as obesity and BMI of around 22 is set as standard physique based on the WHO criteria for obesity. The health risk of obesity is then clarified by comparing the health information between the two. In attempting a new analysis, Takeyama and Fujii [18] applied the wavelet interpolation model to the secular trends in health information of male university students, and constructed a span evaluation chart. We applied this chart to the group of university students established as severely obese with BMI of $\geq 30$, and found that their health information values were fairly low. That is, the health information of subjects classified as severely obese did not show that it was such a large health risk From these findings, the level of risk to health from obesity was ambiguous in comparisons made with severe obesity established as a group.

Therefore, to clarify the level of the risk to health from obesity, more detailed analysis of the composition of obesity level and health information is needed. This study thus explored the composition of trends in health information from fluctuations in BMI, a simple indicator of obesity. The meaning of BMI fluctuations is that they show a detailed transition slide of level of fatness. That is, by sliding BMI one unit at a time, the health information contained in that one unit can be calculated. The health information of 1 unit of BMI has meaning as the rate of change, and more rigorous health information changes can be ascertained. For example, looking at the trend in body fat percentage for one unit of BMI (Figure 6 ), a rising trend with a constant rate is shown. That is, BMI fluctuations are shown with the same precision as body fat percentage, which can ensure the determination of fatness. Then, by analyzing the trends in health information according to the slide in BMI fluctuations, it becomes possible to clarify the level of health risk.

First, looking at systolic blood pressure, blood pressure accompanying BMI fluctuations clearly showed a rising trend. Without confirming the critical point in the elevation of blood pressure, a constant trend toward obesity increased the health risk of elevated blood pressure. Hirohara [19] showed a strong correlation between blood pressure and BMI, and the results of this study are thought to show the same thing. However, an inflection point is seen at BMI of 29.6, and it is suggested from this inflection point that the health risk rises with rapid systolic blood pressure. Iwata et al. [20] reported an increased likelihood of diseases such as cerebral infarction, cerebral hemorrhage, subarachnoid hemorrhage, myocardial infarction, and angina. Based on the finding in this study of an inflection point in blood pressure elevation accompanying BMI fluctuations at BMI of 29.6, this can be said to show a rising health risk by showing marked blood pressure elevations when BMI exceeds 30 . Thus, if we were to show an obesity cutoff point for disease, it may be that it would be BMI of 29.6 (about 30).

Next, with diastolic blood pressure an inflection point was seen at BMI of 19.1. The inflection point seen with diastolic blood pressure is the lower limit, beyond which diastolic blood pressure again rises. The health risk is shown to gradually rise. This trend does not show a rapid health risk beyond the inflection point as is seen with BMI of 29.6 for systolic blood pressure. However, for thinness in particular, a large rise in blood pressure is seen around a BMI of 19.1, which is the lower inflection point. According to Tsuji et al. [21], "thin" people have more activity of the sympathetic nerves, which contract the blood vessels, and there was a tendency for diastolic blood pressure to rise. The results for thinness in this study show the same, and it was clarified that people who tend to be thin have a higher rate of elevation in diastolic blood pressure than those who are obese. Therefore, the cutoff value for health in diastolic blood pressure may be conjectured to be when the inflection point of BMI is 19.1.

With maximal oxygen uptake, an inflection point was seen when BMI was 17.6. Demura et al. [22] demonstrated that motor ability, to which whole body endurance contributes, is lower when body fat percentage is high. Certainly, looking at the change trend in body fat percentage with BMI fluctuations (Figure 6), the body fat percentage is about $10 \%$ when the inflection point of BMI is 17.6, and maximal oxygen uptake shows a peak. Thus, for motor ability, to which whole body endurance contributes, it may be conjectured that the health risk becomes higher when the peak BMI is 17.6. The maximal oxygen intake also showed a decreasing trend around the inflection point. Therefore, the critical point for maximal oxygen intake with respect to motor ability may be conjectured to be when BMI is 17.6.

Today, blood pressure (systolic blood pressure and diastolic blood pressure) can be measured easily at home. Therefore, it could be seen as a simple indicator of health for university students. Showing the cutoff value for transition to illness from BMI fluctuations with this indicator is thus thought to be a valuable finding. Moreover, considering that blood pressure tends to rise with age, the cutoff value of BMI 29.6 may be a true critical point for the risk level at which obesity connotes disease. We may then venture the further suggestion that BMI of 19.1 presents a cutoff value for diseases associated with thinness. Maximal oxygen uptake is rarely related directly to disease, but considering fitness in terms of general endurance, it is also an element of health as physical vitality. Therefore, if BMI of 17.6 is a critical point for maximal oxygen uptake, it 
would seem to be a possible cutoff value for health. Establishing this kind of simple health indicator may be a finding that contributes to the promotion of appropriate health management in university students.

\section{Conclusion}

This study analyzed the composition of the trends in health information by fluctuations in BMI, a simple indicator of obesity, in male university students. The results demonstrated the slide status of detailed health information figures for each unit of BMI. A cutoff value for health could then be established by identifying the inflection point in BMI fluctuations from the slide status of health information based on BMI fluctuations. There are few studies such as this that have analyzed health risks from health information trends by detailed readings of each BMI fluctuation, and it has given very valuable findings. The cutoff values derived from the health risk analysis based on this health information is very much an indicator that shows illness and health risks simply. It has potential to be a straightforward health index for university students. The cutoff value for systolic blood pressure health in this study was BMI of 29.6, so this value requires attention in real obesity. The cutoff value for diastolic blood pressure was BMI of 19.1, and requires attention with regard to thinness. Of course, it also needs to be taken full note of in real obesity. The critical point for maximal oxygen uptake was BMI of 17.6, which could perhaps be considered a cutoff value for health in terms of physical vitality, as an element of physical fitness. This could be a finding that contributes to appropriate health management in university students.

\section{References}

[1] T. Furugouri, "Economics of obesity," Kadokawa Gakugei Shuppan, 2010.

[2] Ministry of Health, Labour and Welfare. "National Health and Nutrition,"

https://www.mhlw.go.jp/stf/seisakunitsuite/bunya/kenkou_iryo u/kenkou/eiyou/h29-houkoku.html, (accessed 2019. 12).

[3] National Center for Health Statistics (NCHS), "overweight and obesity," https://www.cdc.gov/obesity/, (accessed 2019. 12).

[4] A. Matsumoto, "Fundamental Properties of Aldehyde Dehydrogenase 2 (ALDH2) and the Importance of the $A L D H 2$ Polymorphism," Japanese Journal of Hygiene, Vol. 71, No. 1, pp. 55-68, 2016.

[5] M. Asakawa, "Lifestyle Characteristics of Obese people in Terms of Health Checkup Questions," Journal of Japan Society for the Study of Obesity, Vol. 18, p. 171, 2012.

[6] K. Hayakawa, K. Fujii, K. Kasuya, T. Kondoh and N. Tanaka, "The Estimation for Proper Physical Fitness of Company Employees Derived from Body Composition Balance," Production Management, 23 (2), pp. 97-102, 2016.
[7] K. Hayakawa, K. Fujii, K. Kasuya, and N. Tanaka, "Productivity of Defense Education Judged from Optimum Validity of Physical Fitness in Defense Employees," Production Management, 24 (1), pp. 69-74, 2017.

[8] Y. Matsuura and M. Kim, "Analysis of Physical growth by fitting the polynomial to the longitudinal growth distance data of individual - age 6 to 17," Research Monograph issued by Growth and Development Research institute of Health and Sports Sciences, University of Tsukuba, pp. 1-153, 1991.

[9] R. H. Largo, Th. Gasser, A. Prader, W. Stuetzle, and P. J. Huber, "Analysis of the adolescent growth spurt using smoothing spline functions," Annals of Human Biology, Volume 5, pp. 421-434, 1978.

[10] K. Fujii, and Y. Matsuura, "Analysis of the growth velocity curve for height by the Wavelet Interpolation Method in children classified by maturity rate," American Journal of Human Biology, Vol. 11, pp. 13-30, 1999.

[11] K. Fujii, and S. Demura, "Confirmation of Delayed Menarche Based on Regression Evaluation of Age at Menarche for Age at MPV of Height in Female Ball Game Players," Environmental Health and Preventive Medicine, Vol. 10, pp. 48-54, 2005.

[12] K. Fujii, and Y. Yamamoto, "The analysis of the growth velocity curve in height based upon the maturity rate," Japan Journal of Physical Fitness and Sports Medicine, Vol. 44, pp. 431-438, 1996.

[13] A. Quetelet, "Sur I' home et le development de ses faculties," Essai de physique sociale, Bachelier, Pairs, Vol. 2, 1835.

[14] A. Keys, F. Fidanza, M. J. Karvonen, N. Kimura, and H. L. Taylor, "Indices of relative weight and obesity," J Chronic Dis, 25, p329-343, 1972.

[15] Y. Matsuzawa and I. Yasuo, "Definition and diagnostic criteria of metabolic syndrome," Journal of the Japanese Society of Internal Medicine, 94, pp. 794-809, 2005.

[16] K. Fujii, "Essential causal relation of BMI and fat that contributes to female trunk circumference information," AIT The Review of Business Administration and Computer Science, 12 (1), pp. 19-32, 2017.

[17] T. Kuroki, "Kenko, Rouka, Jumyou (Health, Ageing, lifespan)" Chuokoron-Shinsha, 2007.

[18] Y. Takeyama, K. Fujii, Y. Naito, T. Sakai, and K. Hayakawa, "Risk Analysis of Body Balance by Youth Severe Obesity," Production Management, 25 (2), pp. 143-148, 2018.

[19] T. Hirohara and K. Hattori, "Blood Pressure and Related Factors in Male High School Students," Bull. Inst. education, Ibaraki University, Vol. 21, pp. 225-233, 2002.

[20] M. Iwata, K. Takakura, H. Noguchi, S. Matsui, and Y. Yamamoto, "Comparison of blood pressure and lifestyle in the university student of sugitani campus divided according to BMI," Clinical Study of Campus Life, No. 13, pp. 23-26, 2019.

[21] R. Tsuji, Y. Kodaira, T. Oota, and T. Yahata, "Energy metabolism and autonomic regulatory function in lean female students," Journal of Japan Health Medicine Association. 13 (1), pp. 11-17, 2004.

[22] S. Demura, "Health sports science lecture," Tokyo: Kyorin-shoin, 2012. 